\title{
Implementation of the 2021 molecular ESGO/ESTRO/ESP risk groups in endometrial cancer
}

\author{
Sara Imboden b, Denis Nastic a, Mehran Ghaderi a , Filippa Rydberg a , Franziska Siegenthaler ${ }^{\text {b }}$, \\ Michael D. Mueller ${ }^{\mathrm{b}}$, Tilman T. Rau ${ }^{\mathrm{c}}$, Elisabeth Epstein ${ }^{\mathrm{d}}$, Joseph W. Carlson ${ }^{\mathrm{a}, *}$ \\ a Department of Oncology-Pathology, Karolinska Institutet, Department of Pathology and Cytology, Karolinska University Hospital, Stockholm, Sweden \\ ${ }^{\mathrm{b}}$ Department of Obstetrics and Gynecology, Bern University Hospital and University of Bern, Bern, Switzerland \\ ${ }^{\mathrm{c}}$ Institute of Pathology, University of Bern, Bern, Switzerland \\ d Department of Clinical Science and Education, Karolinska Institutet, Department of Obstetrics and Gynecology, Södersjukhuset, Stockholm, Sweden
}

\section{H I G H L I G H T S}

- Application of the $2021 \mathrm{ESGO/ESTRO/ESP} \mathrm{molecular} \mathrm{risk} \mathrm{groups} \mathrm{is} \mathrm{feasible} \mathrm{and} \mathrm{shows} \mathrm{significant} \mathrm{differences} \mathrm{in} \mathrm{survival.}$

- Immunochemistry for TP53 and MMR and applying POLE sequencing is only needed in selected cases.

- The shift in the risk groups are done by P53and and POLEmut classifications in a sizeable number of patients.

\section{A R T I C L E I N F O}

\section{Article history:}

Received 11 March 2021

Accepted 23 May 2021

Available online 11 June 2021

\section{Keywords:}

Endometrial cancer

Genomic subgroups

Risk group

MMR

POLE

P53

\section{A B S T R A C T}

Introduction. In 2021, a joint ESGO/ESTRO/ESP committee updated their evidence-based guidelines for endometrial cancer, recommending a new risk grouping incorporating both clinicopathologic and molecular parameters. We applied the new risk grouping and compared the results to those of the prior 2016 clinicopathologic system.

Materials and methods. We classified molecularly a cohort of 604 women diagnosed with endometrial cancer using immunohistochemistry for TP53 and MMR proteins on a tissue microarray, as well as Sanger sequencing for POLE mutations. These results, combined with clinicopathologic data, allowed the patients to be risk grouped using both the new 2021 molecular/clinicopathologic parameters and the prior 2016 clinicopathologic system.

Results. The application of the 2021 molecular markers shows Kaplan-Meier curves with a significant difference between the groups for all survival. Molecular classification under the 2021 guidelines revealed a total of 39 patients (39/594, 7\%) with a change in risk group in relation to the 2016 classification system: the shift was alone due to either P53abn or POLEmut molecular marker. In order to ensure correct 2021 molecular risk classification, not all patients with endometrial cancer need a molecular diagnostic: $433(72.9 \%)$ cases would need to be analyzed by TP53 IHC, only 46 (7.7\%) by MMR IHC and 286 (48.1\%) POLE sequencing reactions.

Conclusion. Application of the 2021 molecular risk groups is feasible and shows significant differences in survival. IHC for TP53 and MMR and applying POLE sequencing is only needed in selected cases and leads to shifting risk groups both upward and downward for a sizeable number of patients. It is possible to significantly reduce the number of analyses required to implement the classification if resources are limited.

(C) 2021 The Authors. Published by Elsevier Inc. This is an open access article under the CC BY license (http:// creativecommons.org/licenses/by/4.0/).
Abbreviations: EC, endometrial cancer; LVSI, lymphovascular space invasion; POLE, polymerase epsilon; TGCA, The Cancer Genome Atlas; TMA, tissue microarray; OS, overall survival; DSS, disease-specific survival; RFS, recurrence-free survival; IHC, Immunohistochemistry; ESGO, European Society of Gynecological Oncology; ESTRO, European Society for Radiotherapy and Oncology; ESP, European Society of Pathology,; NSMP, No specific molecular profile.

* Corresponding author at: Department of Pathology, Keck School of Medicine at USC, 2011 Zonal Ave., HMR 311A, Los Angeles, CA 90089, USA.

E-mail address: joseph.carlson@med.usc.edu (J.W. Carlson).

\section{Introduction}

In January 2021 the European Society of Gynecological Oncology (ESGO), the European Society for Radiotherapy and Oncology (ESTRO), and the European Society of Pathology (ESP) published updated guidelines for risk group determination in endometrial cancer, integrating both molecular diagnostics and clinicopathologic variables, with the goal of improving patient treatment [1]. These molecular prognostic risk groups represent a revolutionary change in the management of women with endometrial cancer (EC) and will require a total 
reorientation of how these patients are diagnosed and treated. Prior to the issuance of these guidelines, prognostic risk groups were based on postoperative clinicopathologic findings such as tumor histology, stage of disease, grade, and lymphovascular space invasion (LVSI) [2-5]. The new 2021 molecular risk groups are a hybrid of clinicopathologic findings and molecular markers. The new risk grouping serves as the basis for patient management, especially the use and type of adjuvant therapy. However, implementation of the 2021 molecular risk groups carries with it numerous challenges. These include, but are not limited to, (1) introduction of molecular testing for a relatively common tumor that is treated at both specialized cancer centers and smaller, more community-based, centers, (2) interpretation of molecular diagnostic results by clinical teams that may not have encountered them previously, and (3) movement of patients between risk groups in sometimes surprising ways, which may cause worry and concern by the patient and care team.

The molecular understanding of EC has seen an incredible evolution over the past decade. In 2014, The Cancer Genome Atlas (TCGA) [6] developed an integrated genomic classification of endometrial cancer (EC), dividing cases into four genomic subgroups: (1) copy number high "serous-like," (2) copy number low "endometrioid-like," (3) polymerase epsilon (POLE) ultramutated, and (4) microsatellite unstable "MSI (hypermutated)", with each group exhibiting a different progressionfree survival. Since then, numerous research groups have explored ways to introduce a clinically applicable molecular-based classification system for EC [7-15]. These molecular markers have been evaluated in isolation, as well as in classification schema, in order to compare them with traditional clinicopathologic based systems. Finally, in the 5th addition of the WHO Classification of Female Genital Tumors, published in September 2019, the molecular classification is integrated, finalizing the definite place of molecular markers in the diagnosis of EC [16].

The primary objective of the current study was to evaluate the implementation of the 2021 ESGO/ESTRO/ESP molecular risk groups in a large and unselected EC patient cohort. A second objective was to compare the new risk groups with the 2016 clinicopathologic only, non-molecular risk groups. A third objective was to identify challenges to implementation, with the goal of providing concrete guidance to clinics, large and small, implementing this system $[2,17]$.

\section{Materials and methods}

\subsection{Patient cohort and clinical data}

The study was approved by the local ethics boards in Stockholm and in Bern (2016/362 and 2018-00479, respectively); ethics approval for the bio banking of tissue was granted from the regional biobank review board in each country. All women provided written informed consent for the use of their bio banked tissue and clinical data for research purposes.

This is a retrospective cohort study; the women were selected without reference to tumor type and therefore can be said to represent a population-based cohort of all EC women during the years 20042015. A cohort of 349 women diagnosed with EC from Karolinska University Hospital (2011-2015) and 255 women from Bern University Hospital (2004-2015) was established (KImBer cohort). Clinical and radiological data, medical history, and treatment data as well as pathology-associated parameters were obtained through a review of digital medical records and pathology reports. Follow-up data on recurrence and survival are available through standardized databases and follow-up controls in both clinics. All pathology slides were reviewed by reference pathologists (Karolinska cases, DN, JC, and Bern cases, TR) to confirm grade and histotype.

\subsection{Molecular classification: 2021 ESGO/ESTRO/ESP}

Molecular analysis was applied following the WHO Classification of Tumors, 5th Edition, Volume 4: Female Genital Tumors. Cases were classified as POLEmut, MMRd, NSMP, and p53abn [7-10,18-21]). We constructed a TMA (tissue microarray) upon which we performed immunohistochemistry for p53 and MMR proteins MLH1, MSH2, MSH6, and PMS2. Mismatch repair protein deficiency (MMRd) was defined as loss of nuclear staining in at least one out of the four MMR proteins. P53 aberrant protein expression (p53abn) was defined as either complete loss of nuclear protein expression or strong homogenous nuclear overexpression. Detailed description of the methods of staining are under Supplementary materials and Supplementary Fig. 1.

\subsection{POLE sequencing}

To identify POLE (ultramutated) tumors, DNA was isolated from two $1 \mathrm{~mm}$ core punches of formalin-fixed paraffin-embedded tumor tissue using a commercial DNA extraction kit (PerkinElmer chemagen Technology, chemangic protocol name: DNA formalin-fixed paraffinembedded external lysis VD101124.che) according to the manufacturer's protocol. All EC cases were analyzed for mutations of POLE gene (NM.006231) exons 9-14 by Sanger sequencing. Details of primers and interpretation of mutations has been published previously [15]. Mutation analysis was done using Mutation Surveyor ${ }^{\mathrm{TM}}$ software, the Phred Quality Threshold was set to 15 as default. Non-synonymous mutations were confirmed by resequencing and, in the cases of mutations with unknown predicted pathogenicity. DNA from normal tissue (for example, myometrium) was extracted and sequenced for the described mutation in order to exclude germline variants.A tumor was considered POLE mutated (POLEmut) if sequencing proved the existence of a hotspot mutation in the exonuclease domain POLE, as described in our publication on POLEmut tumors [15] and verified by Gilks et al. [22].

\subsection{Multiple classifiers}

Analyzing tumors for $\mathrm{p} 53, \mathrm{MMR}$ and POLE can reveal defects in multiple molecular markers. These tumors were classified as recommended in the 2021 ESGO/ESTRO/ESP system (i.e., first POLEmut, then MMRd, then p53abn).

\subsection{Prognostic risk grouping}

The patients were divided into two prognostic risk groups: (1) addition of the new 2021 ESGO/ESTRO/ESP molecular classification system and (2) the 2016 clinicopathologic system only. This is based on FIGO Stage, Histology, Grading, LVSI and in the new classification 2021 the molecular subtype (POLEmut, p53abn, MMRd or NSMP) is added as defined by the publication 2021 [1].

A flow chart was developed, that allowed an identification of each patient into a risk category by using as few supplementary testing as possible. For this, the groups were selected, where the molecular subtype can change the grouping (Stage 1 and 2 ) and then divided by histology. The minimal use of molecular diagnostic was applied, to be able to correctly annotate each patient to a risk grouping.

\subsection{Statistical analysis}

To address the first objective of our study, prognostic risk groups were examined looking at overall survival (OS), disease-specific survival (DSS), and recurrence-free survival (RFS) using both KaplanMeier plots with log-rank significance testing and Cox proportionalhazards regression models.

Associations between molecular classifiers and other variables such as demographic and clinicopathological factors were tested using nonparametric tests. $t$-test and ANOVA were used for continuous variables; log-rank test, chi-square, and Fisher's exact test were used for categorical variables.

A statistical significance level of 0.05 was used. IBM SPSS software version 24.0 was applied. 


\section{Results}

\subsection{General clinicopathological characteristic}

Initially, 604 women were included in this study. Molecular classification (one or more molecular markers) was not possible for 10 of them; these women were therefore excluded from the analyses. At initial diagnosis, mean and median ages were 66.1/67 (range 31-93) years, and BMI was 29/27.6 (range 16.4-58.6). Only 53 $(8.9 \%)$ of the women were premenopausal at the time of initial diagnosis. Most of the women were either para $2(32.5 \%)$ or nulliparous (23.6\%) (range 0-9).

\subsection{Application of molecular markers}

Results of the molecular classification and associations with clinicopathological characteristics are summarized in Table 1.

Only three clinicopathological factors showed no significant association with the molecular classification (BMI, myometrial invasion and lymph node status: $p$ values of $0.68,0.08$, and 0.2 respectively). Or else the groups differ significantly in their clinical features.

\subsection{ESGO/ESTRO/ESP Molecular Prognostic Risk Group}

Application of the 2021 molecular prognostic risk groups to the cohort revealed the distribution in the five risk groups: low risk $N=243$ (40.9\%), intermediate risk $N=84$ (14.1\%) high-intermediate $N=89$ (15.0\%) high risk $N=148$ (24.9\%) and advanced $N=30$ (5.1\%). A detailed comparison of the 2021 molecular with the 2016 clinicopathologic only prognostic risk groups is shown as a cross table (Table 2).

A total of 39 patients changed group in the new classification. In 17 patients, there is a shift upward due to classification as p53abn. In 22 patients, there is a shift downward due to classification as POLEmut. The shift of patients between prognostic risk groups, as well as the distribution of the molecular subtypes within the prognostic groups, is shown in Fig. 1.

Additionally, three patients show advanced, FIGO Stage 3 disease and POLE mutation. All three patients had hotspot mutations (S297F, V411L and P286R). In the 2021 molecular risk groups, these patients are not clearly classifiable and were left in the high / advanced risk group.

The switch from high risk to low risk and vice versa are the groups that potentially most would affect adjuvant treatment. In this cohort

Table 1

Association between molecular markers, demographic and clinicopathologic factors.

\begin{tabular}{|c|c|c|c|c|c|c|}
\hline \multirow[t]{2}{*}{ Variable } & \multirow[t]{2}{*}{ Total N (\%) } & \multicolumn{5}{|c|}{ Molecular classifier } \\
\hline & & MMRd & POLEmut & P53adn & NSMP & $p$-value association \\
\hline No. Of patients (\%) & $594(100)$ & $199(33.5)$ & $38(6.4)$ & $86(14.5)$ & $271(45.6)$ & \\
\hline \multicolumn{6}{|l|}{ Age } & \multirow[t]{3}{*}{$P=0.001$} \\
\hline Mean $\pm S E$ & $66 \pm 0.4$ & $67.2 \pm 0.7$ & $60.1 \pm 1.4$ & $67 \pm 1$ & $65.8 \pm 0.6$ & \\
\hline Median & 67 & 68 & 60.5 & 67 & 66 & \\
\hline BMI mean \pm SE & $29.1 \pm 0.3$ & $28.5 \pm 0.5$ & $27.3 \pm 1.0$ & $29.3 \pm 0.9$ & $29.7 \pm 0.5$ & \multirow[t]{2}{*}{$p=0.68$} \\
\hline Missing & 106 & 35 & 2 & 18 & 51 & \\
\hline \multicolumn{6}{|l|}{ Grade (\%) } & \multirow[t]{4}{*}{$p=0.00$} \\
\hline G1 & $226(38.0)$ & $59(29.6)$ & $13(34.2)$ & $14(16.3)$ & $140(51.7)$ & \\
\hline G2 & $202(34.0)$ & $88(44.2)$ & $9(23.7)$ & $12(13.9)$ & $93(34.3)$ & \\
\hline G3 & $166(27.9)$ & $52(26.1)$ & $16(42.1)$ & $60(69.8)$ & $38(14.0)$ & \\
\hline \multicolumn{6}{|l|}{ Histotype } & \multirow[t]{3}{*}{$p=0.00$} \\
\hline Non-endo & $100(16.8)$ & $29(14.6)$ & $7(18.4)$ & $48(55.8)$ & $16(5.9)$ & \\
\hline Endometrioid & $494(83.2)$ & $170(85.4)$ & $31(81.6)$ & $38(44.2)$ & $255(94.1)$ & \\
\hline \multicolumn{6}{|l|}{ LVSI } & \multirow[t]{3}{*}{$p=0.00$} \\
\hline Yes & $161(27.1)$ & $62(31.2)$ & $15(39.5)$ & $34(39.5)$ & $50(18.5)$ & \\
\hline No & $433(72.9)$ & $137(68.8)$ & $23(60.5)$ & $52(60.5)$ & $221(81.5)$ & \\
\hline \multicolumn{6}{|l|}{ FIGO Stage } & \multirow[t]{5}{*}{$p=0.00$} \\
\hline I & $443(74.6)$ & $147(73.9)$ & $33(86.8)$ & $49(57.0)$ & $214(79.0)$ & \\
\hline II & $55(9.3)$ & $21(10.6)$ & $2(5.2)$ & $9(10.5)$ & $23(8.5)$ & \\
\hline III & $69(11.6)$ & $27(13.6)$ & $3(7.9)$ & $14(16.3)$ & $25(9.2)$ & \\
\hline \multirow{2}{*}{\multicolumn{6}{|c|}{ Myometrial invasion }} & \\
\hline & & & & & & \multirow{5}{*}{$p=0.08$} \\
\hline intramucosal & $53(8.9)$ & $16(8.0)$ & $1(2.6)$ & $4(4.7)$ & $32(11.8)$ & \\
\hline$<50 \%$ & $305(51.3)$ & $100(50.3)$ & $23(60.5)$ & $39(45.3)$ & $143(52.8)$ & \\
\hline$>50 \%$ & $235(39.6)$ & $82(41.2)$ & $14(36.8)$ & $43(50.0)$ & $96(35.4)$ & \\
\hline Missing & $1(0.2)$ & $1(0.5)$ & 0 & 0 & 0 & \\
\hline \multicolumn{7}{|l|}{ Lymph node status } \\
\hline LN removed & 323 & $84(31.3)$ & $22(8.2 \%)$ & 51(19.0) & $111(41.1)$ & \multirow{3}{*}{$\begin{array}{l}p=0.01 \\
p=0.20\end{array}$} \\
\hline Pos & $63(10.6)$ & $20(10.1)$ & $2(5.3)$ & $17(19.8)$ & $24(8.9)$ & \\
\hline Neg & $236(39.7)$ & $76(38.2)$ & $21(55.3)$ & $41(47.7)$ & $98(36.2)$ & \\
\hline \multicolumn{6}{|l|}{ Adjuvant treat } & \multirow[t]{4}{*}{$p=0.00$} \\
\hline No & $342(57.6)$ & $111(55.8)$ & $22(57.9)$ & $28(32.6)$ & $181(66.8)$ & \\
\hline Yes & $239(40.2)$ & $80(40.2)$ & $16(42.1)$ & $57(66.2)$ & $86(31.7)$ & \\
\hline Missing & $13(2.2)$ & $8(4.0)$ & $0(0)$ & $1(1.2)$ & $4(1.5)$ & \\
\hline \multicolumn{7}{|l|}{ Recurrence } \\
\hline Yes & $88(14.8)$ & $29(14.6)$ & $1(2.6)$ & $26(30.2)$ & $32(11.8)$ & \multirow[t]{3}{*}{$p=0.00$} \\
\hline No & $470(79.1)$ & $152(76.3)$ & $36(94.7)$ & $58(67.4)$ & $224(82.7)$ & \\
\hline Missing & $36(6.1)$ & $18(9.0)$ & $1(2.6)$ & $2(2.3)$ & $15(5.5)$ & \\
\hline Survival & & & & & & \\
\hline Alive & $486(82.1)$ & $162(81.4)$ & $37(97.4)$ & $60(69.7)$ & $227(83.8)$ & \\
\hline DOD & $57(9.6)$ & $15(7.5)$ & $1(2.6)$ & $21(24.4)$ & $20(7.4)$ & $p=0.00$ \\
\hline Other cause & $28(4.7)$ & $9(4.5)$ & 0 & $3(3.5)$ & $16(5.9)$ & \\
\hline Unknown cause & $15(2.5)$ & $9(4.5)$ & 0 & & $6(2.2)$ & \\
\hline Treatment & $3(0.5)$ & $1(0.5)$ & 0 & $2(2.3)$ & 0 & \\
\hline Missing & $3(0.5)$ & $3(1.5)$ & 0 & 0 & $2(0.7)$ & \\
\hline
\end{tabular}


Table 2

Shifting upward and downward of cases between the 2016 and the 2021 molecular risk groups.

\begin{tabular}{|c|c|c|c|c|c|c|}
\hline \multirow[b]{2}{*}{2021 ESGO/ESTRO/ESP Molecular Risk Groups } & \multicolumn{6}{|c|}{2016 Clinicopathologic Risk Groups } \\
\hline & Low risk & Intermed risk & High-intermed risk & High risk & Advanced/metastatic & Total \\
\hline Low risk & 221 & 1 & 9 & 12 & 0 & 243 \\
\hline Intermed risk & 2 & 82 & 0 & 0 & 0 & 84 \\
\hline High-intermed risk & 0 & 0 & 89 & 0 & 0 & 89 \\
\hline High risk & 12 & 1 & 2 & 133 & 0 & 148 \\
\hline Advanced/metastatic & 0 & 0 & 0 & 0 & 30 & 30 \\
\hline Total & 235 & 84 & 100 & 145 & 30 & 594 \\
\hline
\end{tabular}

these were each 12 patients that would with a down switch not get adjuvant treatment such as chemotherapy and 12 patients, where an adjuvant treatment would be indicated.

\subsection{Double and triple classifiers}

In this cohort, 47 patients had tumors with multiple molecular aberrations. There were 45 tumors with double aberrations ( 32 with P53abn and MMRd, 10 with P53abn and POLEmut, 2 with MMRd and POLEmut). There were 2 cases with three aberrations (i.e. P53abn, POLEmut and MMRd).

A total of 126 tumors were P53abn, but of these 10 had a POLEmut and 32 were MMRd ( 2 both). Thus, only 86 tumors were finally classified as P53abn. Similarly, 204 tumors showed MMRd, but of these 5 had a POLEmut. Thus, only 199 tumors were finally classified as MMRd.

Double and triple classifiers influence the shift of cases between 2021 prognostic risk groups. For example, as described above, a case can be placed in the P53abn classification group only if POLEmut and MMRd have been excluded. In this cohort, 10/594 (1.7\%) had both a POLE and p53 mutation, of these 9/10 (90\%) were FIGO stage I or II. Similarly, a case can be placed in the MMRd classification group only if a POLEmut has been excluded. In this cohort, 2/594 (0.3\%) had both a POLEmut and MMRd.

\subsection{Assessment of prognosis}

The 2021 molecular risk groups show a significant difference (all log rank $p=0.000$ ) among the groups when considering recurrence free survival (RFS), disease specific survival (DSS) and overall survival (OS) (Fig. 2).

\subsection{Implementation of molecular diagnostics}

For the 2021 molecular risk grouping, a shift upward is seen only in FIGO Stage I and II tumors classified as P53abn. A shift downward was seen only in tumors classified as POLEmut. Therefore, we tested a model where few supplementary diagnostic tests are applied.

In our study we have $N=498,83.3 \%$ with all FIGO Stage I and II tumors In the endometrioid Histology cohort $(N=43,372.9 \%)$ patients we need immunohistochemistry for p53. In cases showing an aberrant staining pattern (P53abn) ( $N=46,7,7 \%)$, immunohistochemistry is needed for MMRd and sequencing for POLEmut, to allow correct molecular classification.

In order to identify high risk cases that might potentially be shifted downward due to POLEmut classification, an additional 286 (48.1\%) cases would need to be POLE sequenced. These are the nonendometrioid histologies, the P53abn endometrioid and the former intermediate and high risk groups due to FIGO stage, Grade 3 and LVSI positive tumors.

Therefore, as summarized in Fig. 3, in this population based cohort of patients with EC with $N=594$ patients, in $62.9 \%$ need a staining for P53, $7,7 \%$ for MMR Proteins and a total of $N=286(48.1 \%)$ need a sequencing for POLEmut (endometrioid and non-endometrioid) to allow a correct 2021 risk grouping.

\section{Discussion}

In this study, we evaluate the implementation of the 2021 molecular ESGO/ESTRO/ESP prognostic risk groupings in an unselected cohort of endometrial cancer patients. We identify several areas where these groupings differ from their predecessor, and which
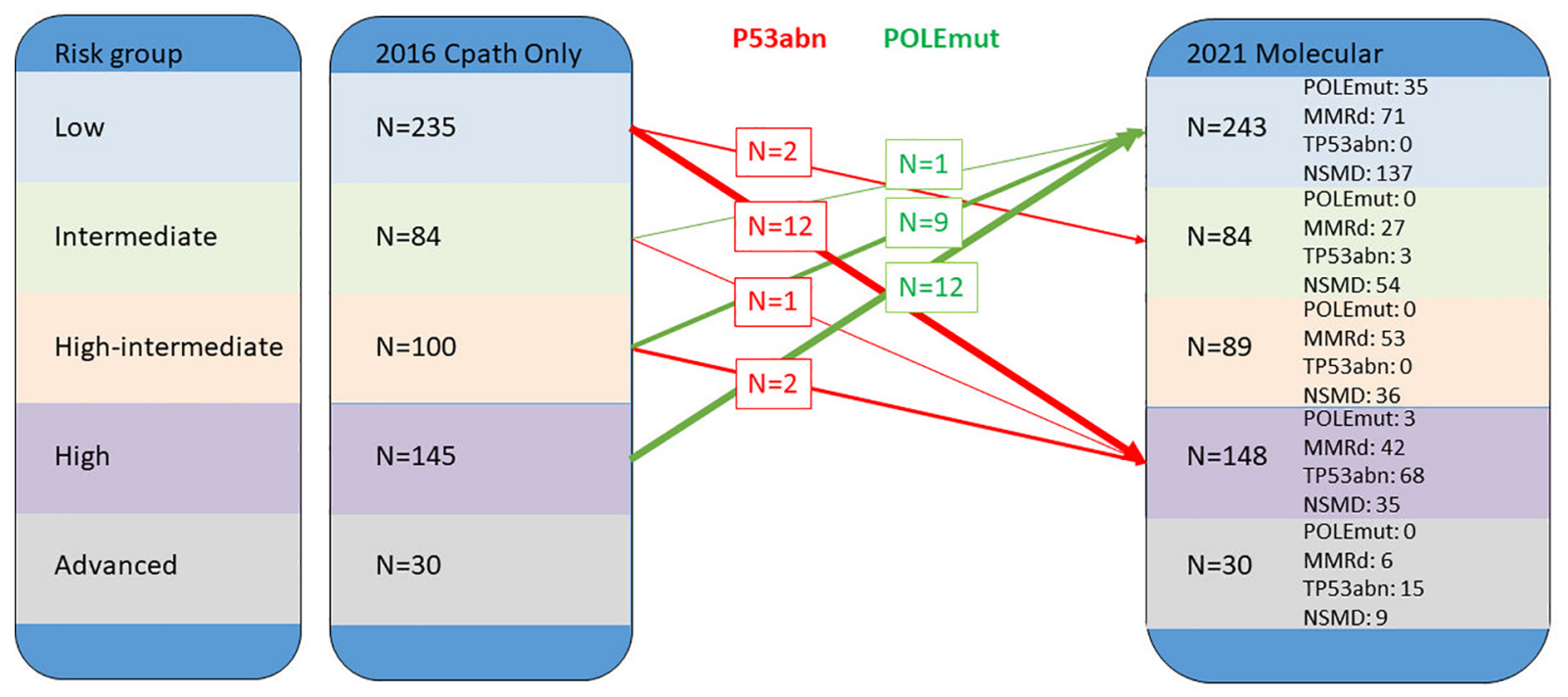

Fig. 1. Change between the old and new risk classification.

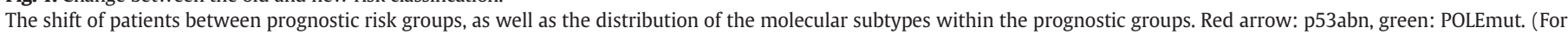
interpretation of the references to colour in this figure legend, the reader is referred to the web version of this article). 

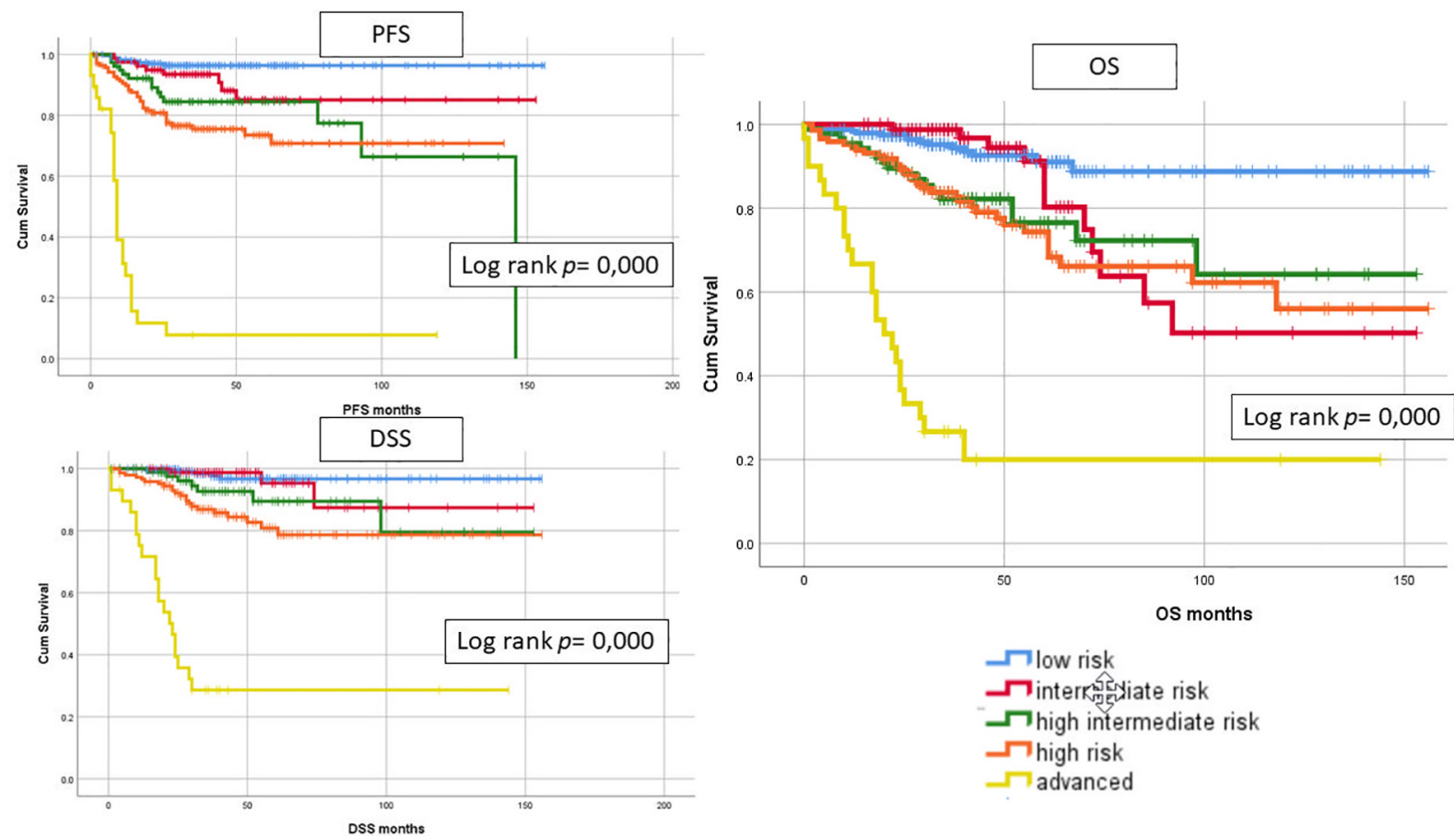

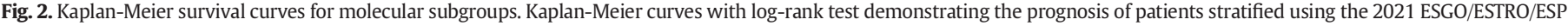
molecular prognostic groups showing Progression free survival (PFS), Disease specific survival (DSS) and overall survival (OS).

may cause difficulty upon implementation. However, it is important, that after implementation gynecologic oncologist must first get familiar with the new set-up and be aware of the aspects, which lead to change in risk grouping.

We can confirm that, the implementation of the 2021 molecular prognostic groupings show significant differences in all survival outcomes, which proves the practicability of the new risk grouping. The last adaption with introduction of LVSI was performed 2016 showing similar results in stratifying the risk groups $[23,24]$. Implementation of the 2021 molecular risk groups on the cohort reveals several important aspects.
First, not all P53abn cases are non endometrioid. Indeed, 26 endometrioid FIGO Grade 1 and 2 (low-grade) cases were identified, which would be improperly classified if molecular markers had not been applied. Are all MMRd cases endometrioid? Even here, 29 MMRd cases were judged to be non endometrioid. The results indicate that using histologic subtype to attempt to "triage" cases to molecular subgroups may lead to significant problems with classification and suggest that markers should be applied independent of histology, which was also confirmed in the resent publication by Weigelt et al. [25]. Applying TP53 immunohistochemistry on all cases of endometrioid FIGO 1 and 2 would represent a significant change for most pathology labs [26].

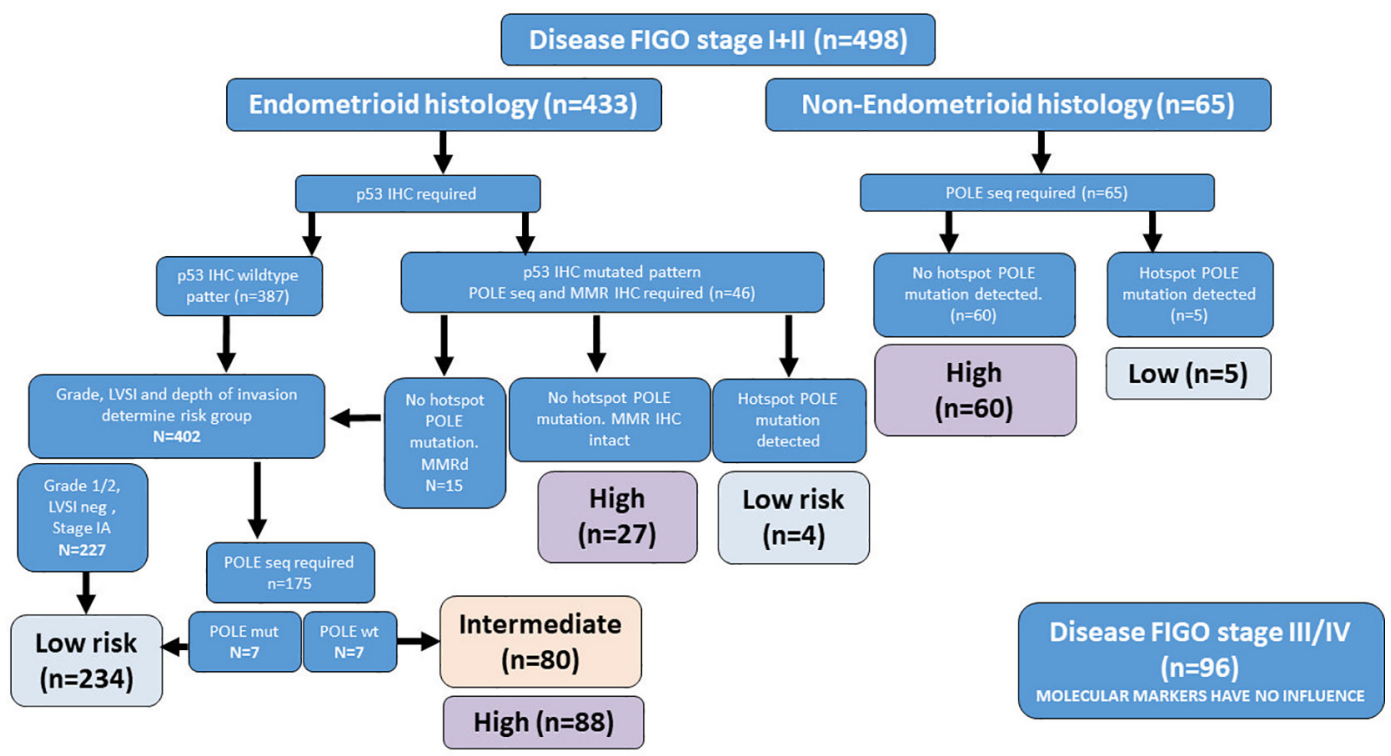

Fig. 3. Flow chart with selective analysis for molecular testing.

Flow chart, which shows how the patients can be triaged to allow testing for P53, MMR or POLE only when indicated. 
In our cohort, fully one-third (26/80,33\%) of P53abn cases would have been missed if endometrioid grade 1 and 2 cases were not stained. Similarly, 15\% (29/199) of MMRd cases would have been missed if only endometrioid cases were evaluated for MMRd. Finally, it is important for labs to have access to POLE and MMR analyses, because assignment to the TP53 molecular group should occur only if POLEmut and MMRd have been excluded. Similarly, assignment to MMRd requires that a POLEmut has been excluded. In our algorithm we could show, that not in all cases a molecular diagnostic is needed, rather only in FIGO Stage I and II. By applying IHC for P53 in 65\% and POLE sequencing in less than $20 \%$ we found a model that is probably more practicable than performing a next generation sequencing for all patients with EC, especially since EC is often not only treated in specialized centers. By applying this model probably also smaller centers can apply the molecular markers in a feasible way.

Comparing the 2016 clinicopathologic and the 2021 molecular risk classification reveals several interesting aspects. First, $8 \%$ of high-risk cases (12/145) downshifted to low-risk after molecular classification due to POLEmut. These cases are specifically mentioned in the recommendations as "rare", but in our cohort they represented the most prevalent reason for risk group shifting [22,27]. In the PORTEC-3 trial, POLEmut stage 3 patients had an excellent outcome, but all were treated with external beam radiotherapy. Second, $5 \%$ of low-risk cases (12/235) shifted upward to high-risk after molecular classification, due to P53abn. Given that low-risk disease is the most common, this means that potentially 1 out of 20 low-risk cases may be misclassified, and subsequently undertreated, without molecular classification. Again, PORTEC-3 indicates that combined therapy leads to a statistically significant survival advantage for P53abn stage 1-3 [28]. These findings and shift in risk group are new and have to be first identified and understood, but then how to manage these cases will probably stay a challenge until we have more information on tailoring adjuvant treatment based on the molecular markers. Finally, note that the 2021 recommendations make a difference between P53abn with and without myometrial invasion [1].

Another important aspect of the risk grouping is, that FIGO Stage, meaning tumor extend including lymph node status remains the most dominant factor: In FIGO Stage >II the molecular marker does not play any role. Therefore, this information (as well as LVSI) stay important for the risk grouping and this information is only available after surgery.

Is it necessary to identify MMRd patients if it does not contribute to their risk grouping? There are several motivations for identifying MMRd patients beyond risk classification, including the desire to identify patients with Lynch syndrome. This is a worthy and important goal for reasons outlined in numerous publications [30,31]. However, from the standpoint of risk classification, MMRd is necessary to correctly classify patients as P53abn. It is important to keep the motivation for the testing clear, so that clinical genetics is consulted and involved in these changes in practice.

To provide concrete guidance for the application of the molecular prognostic risk grouping we show in Fig. 3 possible testing strategy: Evaluating the minimum testing regime necessary to correctly classify patients revealed that correct classification could be achieved in this cohort with 433/594 (72.9\%) TP53 immunohistochemistry, 46/594 (7.7\%) MMR immunohistochemistry, and 286 (48.1\%) POLEmut analyses. Note that additional MMRd analyses may be useful for adjuvant therapy if immunotherapy is potentially indicated.

It is important to realize, that the changes are only in the nonadvanced group and therefore the survival curves stay similar comparing 2016 and 2021. Possibly the major advances of the updated classifiers is the inclusion of additional molecular information to aide therapy decisions (such as immunotherapy) going forward. However, for the adaption of the adjuvant treatment to the molecular findings further prospective studies are still needed.

\section{Conclusion}

Application of the 2021 molecular risk groups is feasible and shows significant differences in survival. The 2021 ESGO/ESTRO/ESP molecular risk groups represent a fundamental change in the clinical diagnosis and treatment of endometrial cancer. Implementing and evaluating these changes is a complex task that is only just beginning. Compared to the 2016 clinicopathologic risk groups, 3.7\% of high-risk patients were shifted downward to low-risk due to a POLE mutation, and $2.9 \%$ of low-risk patients were shifted upward to high risk due to a P53abn classification. This shows that the change is for a sizable number of patients. It is possible to significantly reduce the number of analyses required to implement the classification if resources are limited. Finally, it is important to note that the P53abn group requires exclusion of POLEmut and MMRd, making it distinct from prior descriptions of TP53 mutated tumors (where these exclusions were not made). Although no cases were shifted upward or downward due to MMRd, this analysis is useful in identifying women with Lynch syndrome or as a marker for immunotherapy.

\section{Authors' contributions}

SI, EE, DN, TTR and JWC: Conceptualization, Data curation FR, FS and SI: Validation, SI, DO, DN and JWC: Formal analysis, MG: Methodology, all authors: Writing.

\section{Declaration of Competing Interest}

JWC has received funding from ThermoFisher Scientific/Affymetrix for a different study.

The authors have stated explicitly that there is otherwise no conflict of interest in connection with this article.

\section{Acknowledgments}

The funders had no role in study design, data collection and analysis, decision to publish, or preparation of the manuscript. Funding sources are: Bernese Cancer League (https://bern.krebsliga.ch/), Swiss National Science Foundation (IZSE70_177073)http://www.snf.ch/; Avtal om Läkarutbildning och Forskning (ALF), ALF-Stockholm County (grant no 550411) https://ki.se, Cancer research funding from 'Radiumhemmet' Stockholm, Sweden (grant no 154112) https://www.rahfo.se, Magnus Bergvalls Stiftelse, Cancerfonden, and Foundation for clinicalexperimental cancer research.

\section{Appendix A. Supplementary data}

Supplementary data to this article can be found online at https://doi. org/10.1016/j.ygyno.2021.05.026.

\section{References}

[1] N. Concin, X. Matias, I. Vergote, D. Cibula, M.R. Mirza, S. Marnitz, J. Ledermann, T. Bosse, C. Chargari, A. Fagotti, ESGO/ESTRO/ESP Guidelines for the Management of patients With Endometrial Carcinoma, 2021 12-39, https://doi.org/10.1136/ijgc2020-002230

[2] N. Colombo, C. Creutzberg, F. Amant, T. Bosse, A. González-Martín, J. Ledermann, C. Marth, R. Nout, D. Querleu, M.R. Mirza, C. Sessa, ESMO-ESGO-ESTRO Endometrial Consensus Conference Working Group, ESMO-ESGO-ESTRO Consensus Conference on Endometrial Cancer: diagnosis, treatment and follow-up, Ann. Oncol. Off. J. Eur. Soc. Med. Oncol. 27 (2016) 16-41, https://doi.org/10.1093/annonc/mdv484.

[3] S. Bendifallah, G. Canlorbe, P. Collinet, E. Arsène, F. Huguet, C. Coutant, D. Hudry, O. Graesslin, E. Raimond, C. Touboul, E. Daraï, M. Ballester, Just how accurate are the major risk stratification systems for early-stage endometrial cancer? Br. J. Cancer 112 (2015) 793-801, https://doi.org/10.1038/bjc.2015.35.

[4] S. Bendifallah, G. Canlorbe, E. Raimond, D. Hudry, C. Coutant, O. Graesslin, C. Touboul, F. Huguet, A. Cortez, E. Daraï, M. Ballester, A clue towards improving the European Society of Medical Oncology risk group classification in apparent early 
stage endometrial cancer? Impact of lymphovascular space invasion, Br. J. Cancer 110 (2014) 2640-2646, https://doi.org/10.1038/bjc.2014.237.

[5] T.W. Kong, S.-J. Chang, J. Paek, Y. Lee, M. Chun, H.-S. Ryu, Risk group criteria for tailoring adjuvant treatment in patients with endometrial cancer: a validation study of the gynecologic oncology group criteria, J. Gynecol. Oncol. 26 (2015) 32, https://doi. org/10.3802/jgo.2015.26.1.32.

[6] Cancer Genome Atlas Research Network, C. Kandoth, N. Schultz, A.D. Cherniack, R. Akbani, Y. Liu, H. Shen, A.G. Robertson, I. Pashtan, R. Shen, C.C. Benz, C. Yau, P.W. Laird, L. Ding, W. Zhang, G.B. Mills, R. Kucherlapati, E.R. Mardis, D.A. Levine, Integrated genomic characterization of endometrial carcinoma, Nature 497 (2013) 67-73, https://doi.org/10.1038/nature12113.

[7] A. Talhouk, M.K. McConechy, S. Leung, W. Yang, A. Lum, J. Senz, N. Boyd, J. Pike, M. Anglesio, J.S. Kwon, A.N. Karnezis, D.G. Huntsman, C.B. Gilks, J.N. McAlpine, Confirmation of ProMisE: a simple, genomics-based clinical classifier for endometrial cancer, Cancer. 123 (2017) 802-813, https://doi.org/10.1002/cncr.30496.

[8] A. Talhouk, L.N. Hoang, M.K. McConechy, Q. Nakonechny, J. Leo, A. Cheng, S. Leung, W. Yang, A. Lum, M. Köbel, C.H. Lee, R.A. Soslow, D.G. Huntsman, C.B. Gilks, J.N. McAlpine, Molecular classification of endometrial carcinoma on diagnostic specimens is highly concordant with final hysterectomy: earlier prognostic information to guide treatment, Gynecol. Oncol. 143 (2016) 46-53, https://doi.org/10.1016/j. ygyno.2016.07.090.

[9] A.N. Karnezis, S. Leung, J. Magrill, M.K. McConechy, W. Yang, C. Chow, M. Kobel, C.-H. Lee, D.G. Huntsman, A. Talhouk, F. Kommoss, C.B. Gilks, J.N. McAlpine, Evaluation of endometrial carcinoma prognostic immunohistochemistry markers in the context of molecular classification, J. Pathol. Clin. Res. 3 (2017) 279-293, https://doi.org/ $10.1002 /$ cjp2.82.

[10] A. Talhouk, M.K. McConechy, S. Leung, H.H. Li-Chang, J.S. Kwon, N. Melnyk, W. Yang, J. Senz, N. Boyd, A.N. Karnezis, D.G. Huntsman, C.B. Gilks, J.N. McAlpine, A clinically applicable molecular-based classification for endometrial cancers, Br. J. Cancer 113 (2015) 299-310, https://doi.org/10.1038/bjc.2015.190.

[11] S. Kommoss, M.K. McConechy, F. Kommoss, S. Leung, A. Bunz, J. Magrill, H. Britton, F. Kommoss, F. Grevenkamp, A. Karnezis, W. Yang, A. Lum, B. Krämer, F. Taran, A. Staebler, S. Lax, S.Y. Brucker, D.G. Huntsman, C.B. Gilks, J.N. McAlpine, A. Talhouk, Final validation of the ProMisE Molecular classifier for endometrial carcinoma in a large population-based case series, Ann. Oncol. Off. J. Eur. Soc. Med. Oncol. (2018) https://doi.org/10.1093/annonc/mdy058.

[12] L. Vermij, V. Smit, R. Nout, T. Bosse, Incorporation of molecular characteristics into endometrial cancer management, Histopathology. 76 (2020) 52-63, https://doi. org/10.1111/his.14015

[13] E. Stelloo, T. Bosse, R.A. Nout, H.J. Mackay, D.N. Church, H.W. Nijman, A. Leary, R.J. Edmondson, M.E. Powell, E.J. Crosbie, H.C. Kitchener, L. Mileshkin, P.M. Pollock, V.T. Smit, C.L. Creutzberg, Refining prognosis and identifying targetable pathways for high-risk endometrial cancer; a TransPORTEC initiative, Mod. Pathol. 28 (2015) 836-844, https://doi.org/10.1038/modpathol.2015.43.

[14] J. McAlpine, A. Leon-Castillo, T. Bosse, The rise of a novel classification system for endometrial carcinoma; integration of molecular subclasses, J. Pathol. (2018)https:// doi.org/10.1002/path.5034.

[15] S. Imboden, D. Nastic, M. Ghaderi, F. Rydberg, T.T. Rau, M.D. Mueller, E. Epstein, J.W. Carlson, Phenotype of POLE-Mutated Endometrial Cancer, PLoS One (2019)https:// doi.org/10.1371/journal.pone.0214318.

[16] E. Oliva, A. Cheung, S. Lax, WHO Classification of Tumours of the Female Genital Tract, 2020.

[17] N. Colombo, E. Preti, F. Landoni, S. Carinelli, A. Colombo, C. Marini, C. Sessa, Endometrial cancer: ESMO clinical practice guidelines for diagnosis, treatment and followup, Ann. Oncol. 24 (2013)https://doi.org/10.1093/annonc/mdt353.

[18] D.N. Church, E. Stelloo, R.A. Nout, N. Valtcheva, J. Depreeuw, N. ter Haar, A. Noske, F. Amant, I.P.M.M. Tomlinson, P.J. Wild, D. Lambrechts, I.M. Jürgenliemk-Schulz, J.J. Jobsen, V.T.H.B.M. Smit, C.L. Creutzberg, T. Bosse, Prognostic significance of POLE proofreading mutations in endometrial cancer, J. Natl. Cancer Inst. 107 (2015) 402, https://doi.org/10.1093/jnci/dju402.
[19] E. Stelloo, R.A.A. Nout, E.M.M. Osse, I.J. Jürgenliemk-Schulz, J.J.J. Jobsen, L.C.C. Lutgens, E.M.M. van der Steen-Banasik, H.W.W. Nijman, H. Putter, T. Bosse, C.L.L. Creutzberg, V.T.H.B.M.T.H.B.M. Smit, Improved risk assessment by integrating molecular and clinicopathological factors in early-stage endometrial cancer - combined analysis of PORTEC cohorts, Clin. Cancer Res. 22 (2016)https://doi.org/10.1158/ 1078-0432.CCR-15-2878.

[20] C.M. Cosgrove, D.L. Tritchler, D.E. Cohn, D.G. Mutch, C.M. Rush, H.A. Lankes, W.T. Creasman, D.S. Miller, N.C. Ramirez, M.A. Geller, M.A. Powell, F.J. Backes, L.M. Landrum, C. Timmers, A.A. Suarez, R.J. Zaino, M.L. Pearl, P.A. DiSilvestro, S.B. Lele, P.J. Goodfellow, P. Goodfellow, An NRG oncology/GOG study of molecular classification for risk prediction in endometrioid endometrial cancer HHS public access, Gynecol. Oncol. 148 (2018) 174-180, https://doi.org/10.1016/j.ygyno.2017.10.037.

[21] E. Stelloo, T. Bosse, R.A. Nout, H.J. MacKay, D.N. Church, H.W. Nijman, A. Leary, R.J. Edmondson, M.E. Powell, E.J. Crosbie, H.C. Kitchener, L. Mileshkin, P.M. Pollock, V.T. Smit, C.L. Creutzberg, Refining prognosis and identifying targetable pathways for high-risk endometrial cancer; a TransPORTEC initiative, Mod. Pathol. 28 (2015) 836-844, https://doi.org/10.1038/modpathol.2015.43.

[22] A. León-Castillo, H. Britton, M.K. McConechy, J.N. McAlpine, R. Nout, S. Kommoss, S.Y. Brucker, J.W. Carlson, E. Epstein, T.T. Rau, T. Bosse, D.N. Church, C.B. Gilks, Interpretation of somatic POLE mutations in endometrial carcinoma, J. Pathol. 250 (2020) 323-335, https://doi.org/10.1002/path.5372.

[23] S. Bendifallah, L. Ouldamer, V. Lavoue, G. Canlorbe, E. Raimond, C. Coutant, O. Graesslin, C. Touboul, P. Collinet, E. Daraï, M. Ballester, Patterns of recurrence and outcomes in surgically treated women with endometrial cancer according to ESMO-ESGO-ESTRO consensus conference risk groups: results from the FRANCOGYN study group, Gynecol. Oncol. 144 (2017) 107-112, https://doi.org/ 10.1016/j.ygyno.2016.10.025.

[24] N. Colombo, C. Creutzberg, F. Amant, T. Bosse, A. González-Martín, J. Ledermann, C. Marth, R. Nout, D. Querleu, M.R. Mirza, C. Sessa, ESMO-ESGO-ESTRO consensus conference on endometrial Cancer: diagnosis, treatment and follow-up, Ann. Oncol. Off. J. Eur. Soc. Med. Oncol. 27 (2016) 16-41, https://doi.org/10.1093/annonc/mdv484.

[25] A. Da, C. Paula, D.F. Delair, L. Ferrando, D.J. Fix, R.A. Soslow, K.J. Park, S. Chiang, J.S. Reis-filho, A. Zehir, M.T.A. Donoghue, M. Wu, D.N. Brown, R. Murali, C.F. Friedman, D. Zamarin, V. Makker, J.J. Mueller, M.M. Leitao, N.R. Abu-rustum, C. Aghajanian, B. Weigelt, Gynecologic oncology genetic and molecular subtype heterogeneity in newly diagnosed early- and advanced-stage endometrial cancer, Gynecol. Oncol. (2021)https://doi.org/10.1016/j.ygyno.2021.02.015.

[26] A. Manuscript, P. Onlinefirst, Downloaded from clincancerres.aacrjournals.org on March 11, 2021. (C) 2021 American Association for Cancer Research, 2021https:// doi.org/10.1158/1078-0432.CCR-20-4436.

[27] D.N. Church, E. Stelloo, R.A. Nout, N. Valtcheva, J. Depreeuw, N. Ter Haar, A. Noske, F. Amant, I.P.M. Tomlinson, P.J. Wild, D. Lambrechts, I.M. Jurgenliemk-Schulz, J.J. Jobsen, V.T.H.B.M. Smit, C.L. Creutzberg, T. Bosse, Prognostic significance of POLE proofreading mutations in endometrial cancer, J. Natl. Cancer Inst. 107 (2015) https://doi.org/10.1093/jnci/dju402.

[28] S.M. De Boer, M.E. Powell, L.R. Mileshkin, H.J. Mackay, A. Leary, H.W. Nijman, N. Singh, P.M. Pollock, P. Bessette, A. Fyles, C. Haie-meder, V.T.H.B.M. Smit, R.J. Edmondson, H. Putter, H.C. Kitchener, E.J. Crosbie, M. De Bruyn, R.A. Nout, N. Horeweg, C.L. Creutzberg, T. Bosse, Molecular Classi fi cation of the PORTEC-3 Trial for High-Risk Endometrial Cancer: Impact on Prognosis and Bene fi t From Adjuvant Therapy Abstract, 38, 2021https://doi.org/10.1200/JCO.20.00549.

[30] A.M. Mills, E.A. Sloan, M. Thomas, S.C. Modesitt, M.H. Stoler, K.A. Atkins, C.A. Moskaluk, Clinicopathologic Comparison of Lynch Syndrome - associated and “ Lynch-like " Endometrial Carcinomas Identified on Universal Screening Using Mismatch Repair Protein Immunohistochemistry, 40, 2016 155-165.

[31] C. Stinton, H. Fraser, L. Al-khudairy, R. Court, M. Jordan, D. Grammatopoulos, S. Taylor-phillips, Gynecologic oncology testing for lynch syndrome in people with endometrial cancer using immunohistochemistry and microsatellite instability-based testing strategies - a systematic review of test accuracy, Gynecol. Oncol. 160 (2021) 148-160, https://doi.org/10.1016/j.ygyno.2020.10.003. 